\title{
A influência da resposta dos EUA à COVID-19 no contexto da Saúde Global
}

\author{
The influence of the U.S. response to COVID-19 in Global Health
}

Fabius Vieira Leineweber (https://orcid.org/0000-0003-4151-449X) ${ }^{1}$

Jorge Antonio Zepeda Bermudez (https://orcid.org/0000-0002-4657-0709) ${ }^{2}$
${ }^{1}$ Instituto de Tecnologia em Fármacos, Farmanguinhos, Fundação Oswaldo Cruz (Fiocruz). R. Sizenando Nabuco 100, Manguinhos. 21041-250 Rio de Janeiro RJ Brasil. fabius. leineweber@far.fiocruz.br ${ }^{2}$ Escola Nacional de Saúde Pública Sérgio Arouca, Fiocruz. Rio de Janeiro RJ Brasil.

\begin{abstract}
The American response to the pandemic involves a prominent volume of federal resources, especially for developing and acquiring products for internal use, such as diagnostics or vaccines. Investment mechanisms and historical aspects justify this expenditure. Thus, the social construction of nationalism in American society hinders access to health technologies. The review of such aspects shows how the United States (U.S.) secured a large number of potential products, ensuring excessive local production. This unilateral foreign policy has influenced other countries or regional blocs and undermined global cooperation and solidarity, affecting the collective health of several nations.

Key words COVID-19, Health Sciences, Technology, and Innovation Management, Government Agencies, Access to Technological Innovation
\end{abstract}

Resumo A resposta americana à pandemia envolve um proeminente volume de recursos federais, em especial destinados ao desenvolvimento $e$ aquisição de produtos no uso interno, como diagnósticos ou vacinas. As justificativas para esse desembolso se baseiam em mecanismos de investimentos e aspectos históricos. Assim, a construção social do nacionalismo na formação na sociedade americana prejudica o acesso a tecnologias em saúde. A revisão desses aspectos demonstra como os Estados Unidos (EUA) garantiram compra de grande quantitativo de produtos em potencial, inclusive assegurando excessiva produção local. Essa politica externa unilateral tem influenciado outros países ou blocos regionais e prejudicado a cooperação e a solidariedade global com impacto na saúde coletiva de diversas nações.

Palavras-chave COVID-19, Gestão de Ciência, Tecnologia e Inovação em Saúde, Órgãos Governamentais, Acesso à Inovação Tecnológica 


\section{Introdução}

Com mais de um milhão de mortos, e ainda sem um produto como resposta efetiva à pandemia, o mundo se divide entre esforços internacionais e domésticos. Os tratamentos não estarão disponíveis imediatamente, e a competição por eles pode prejudicar o acesso. $\mathrm{O}$ equilíbrio entre velocidade e eficácia no desenvolvimento também é um desafio econômico. A eficiência da captura dos investimentos financeiros tem componentes humanitários ${ }^{1}$ confrontados por interesses políticos $^{2}$. Embora a atenção coletiva às vacinas seja maior, outros produtos como diagnósticos e terapias são essenciais, além das intervenções não farmacológicas.

Mesmo que as tecnologias em saúde para combate à COVID-19 fossem consideradas bens públicos globais, o acesso não estaria garantido, pois esse conhecimento precisaria ser produzido e distribuído. Nesse sentido, para além do Access to COVID-19 Tools Accelerator (ACT-Accelerator) e o COVID-19 Technology Access Pool (C-TAP), com base nas ações da Global Alliance for Vaccines and Immunization (GAVI), a Organização Mundial da Saúde (OMS) lançou uma estratégia para a produção de vacinas com distribuição eficiente pelo mundo, denominada Covax. Não obstante, ao mesmo tempo que investem para uma resposta global solidária e cooperativa, países como Alemanha, França, Holanda e Itália fazem compromissos de compra próprios, e o Reino Unido também tem investido de forma independente ${ }^{3}$.

Os Estados Unidos (EUA) não participam da Covax; mesmo assim, suas medidas nacionalistas para resposta à COVID-19 dispõem de um volume de recursos financeiros superior aos mecanismos cooperativos globais, encontrando lastro no sistema global de comércio, referenciado na moeda americana. Isso revela que a preponderância de interesses nacionais na política externa americana não é recente. No entanto, a atuação dos EUA na liderança global apresenta mudanças. Novos mecanismos de financiamento executam as operações de fomento e compras antecipadas. Embora comparativamente a outros países, os valores compromissados pelos EUA sejam muito altos, o montante não apresenta discrepância relevante como parcela do PIB americano, e apresenta proporcionalidade com um elevado histórico de despesas públicas em saúde nos EUA, fomentando um grande ecossistema de inovação, apesar das inequidades.

O objetivo do presente artigo é, ao analisar a resposta à pandemia dos EUA, alertar para os riscos para a Saúde Global com o potencial comprometimento mundial no abastecimento e acesso a tecnologias, que podem impactar o Brasil. O breve percurso histórico descrito evidencia a coerência dos posicionamentos atuais com a construção contemporânea do nacionalismo norte-americano. Os investimentos dos EUA nos programas de enfrentamento à COVID-19 revelam elevadas somas de recursos: na pesquisa, no desenvolvimento, na infra-estrutura e nos insumos tecnológicos - respaldados por mecanismos institucionais e culturais que se destacam na disputa pelas vacinas em desenvolvimento. Esses aspectos influenciam outros países ou regiões, reduzem o potencial de solidariedade implementadas pelos organismos multilaterais, prejudicando dessa forma o acesso de países às tecnologias em desenvolvimento, necessárias para uma resposta efetiva dada a interconectividade atual do mundo.

\section{Metodologia}

O artigo apresenta uma revisão do nacionalismo americano e enfrentamentos dos EUA às pandemias recentes, e como isso influenciou na atual resposta à COVID-19. A narrativa não utiliza critérios explícitos e sistemáticos para análise da literatura. Foram pesquisadas fontes secundárias nas páginas eletrônicas das instituições citadas ao longo do texto, que apresentam os valores de investimentos americanos em pandemias anteriores assim como o quantitativo ao longo do tempo dos investimentos recentes, classificado por tecnologia ou etapas de pesquisa, desenvolvimento ou produção. O artigo também acompanha o registro dos eventos relacionados com a pandemia, nas esferas internas nos EUA e a repercussão externa. A partir dessas informações, duas categorias se apresentam mais relevantes: os mecanismos de investimentos e o nacionalismo, sendo o excepcionalismo americano um caso particular.

O excepcionalismo se apresenta dentro de um contexto histórico construtivista, com elementos políticos e econômicos. Esse conceito, de uso frequente nas relações internacionais, conjuga as categorias que direcionam as instituições com base no estudo de caso de resposta à pandemia. Dessa forma, a alocação de meios no repertório institucional americano repercute na Saúde Global. E a importância para a Saúde Coletiva se delimita pela interface da produção biomédica como determinante de inequidades em saúde que podem afetar o Brasil. 
Os recursos para a pandemia têm sido motivo de competição entre os países. Conforme aumentava a demanda por produtos relacionados à pandemia, a limitação de oferta imediata dos produtos acarretava escassez e elevação de preços ${ }^{4}$. Isso aconteceu para equipamentos de proteção individual, insumos farmacêuticos, equipamentos e continua em novas terapias ou no caso das vacinas promissoras. Como resultado desse aspecto competitivo, a pandemia apresenta potencial de mudança na forma como o financiamento da pesquisa e desenvolvimento de produtos médicos e a respectiva aquisição dos mesmos ocorre.

\section{Mecanismos de investimentos e precificação na pandemia}

No caso dos EUA, os principais mecanismos observados durante a pandemia foram a precificação por valor, compartilhamento de custo e compromissos avançados de mercado. Em especial, o setor farmacêutico se caracteriza por atuar na inovação (incremental e radical) com elevados custos de pesquisa e desenvolvimento e de marketing. No entanto, a forma como os novos produtos são precificados geralmente tem como base, não apenas os custos de produção, mas referências externas de outros produtos ou benefícios associados. Antes mesmos da pandemia, havia discussão sobre diversos novos mecanismos de precificação em medicamentos, entre eles o preço por valor (value-based pricing) ${ }^{5}$. Nesse caso, são levadas em conta os custos evitados pela administração de determinado medicamento ou terapia. Esse critério foi aplicado em outras epidemias e recentemente para medicamentos inovadores para hepatite, câncer ou doenças raras.

Quando existe a possibilidade de ganhos pelo contratado, o compartilhamento de custos é determinado pela legislação federal dos EUA. A parcela de compartilhamento é negociada e depende de proveitos, direitos de propriedade ou interesse nacional ${ }^{6}$. No caso dos investimentos americanos para desenvolvimentos de produtos COVID-19, isso antecede o uso efetivo e se relaciona com a superação de metas. Dessa forma, alguns acordos envolvem preços menores com esse investimento, enquanto outros se limitam à compra. Com o objetivo de criar um mercado para o desenvolvimento de novos produtos, os Advanced Market Commitments (AMC) foram propostos como forma de incentivo, através de um compromisso de compra por determinado valor ${ }^{7}$. Inicialmente aplicado para o desenvolvimento de uma vacina pneumocócica pela Global Alliance for Vaccines and Immunization (GAVI), esse mecanismo expandiu a disponibilidade e o acesso à vacina por países em desenvolvimento, viabilizado pelo suporte global de vários países e do Banco Mundial.

Os Estados Unidos ainda dispõem de mecanismos diferenciados de contratos de compras, denominados Other Transaction Agreements $(\mathrm{OTA})^{8}$. A legislação americana para mecanismos de assistência financeira em acordos de cooperação assegura direitos de propriedade intelectual aos investimentos governamentais. Nesse sentido, o Bayh-Dole Act define direitos específicos de uso da propriedade intelectual: sem autorização, com a devida compensação financeira ou, no caso de provisão de interesse público, em termos razoáveis. Por sua vez, os OTAs estão isentos desse aspecto, são negociados livremente, e consequentemente apresentam riscos. Outro mecanismo de empenho são contratos indefinidos, que permitem início imediato da prestação sem termos definidos. Até julho de 2020, para COVID-19, o governo americano tinha US\$ 2,2 bilhões em contratos indefinidos e US\$ 6,5 bilhões do Departamento de Defesa em OTAs para protótipos e produção'.

\section{Construção contemporânea \\ do nacionalismo americano}

Apesar dos países estarem competindo por recursos para lidar com a pandemia, também existem componentes políticos valorizados. Nesse aspecto, o nacionalismo atual toma importância semelhante a acontecimentos durante a Guerra Fria, como a corrida espacial ou armamentista. No caso americano, o nacionalismo já estava em voga nas propostas do governo atual antes da pandemia, embora conflitos entre globalização e nacionalismo remetam a diversos períodos anteriores. A mudança recente ocorre na forma como os EUA abandonam o papel de liderança global em um multilateralismo assertivo.

Historicamente, movimentos nacionalistas ocorrem nos EUA desde sua fundação, como o Tea Party em revolta às taxas britânicas. Portanto, o seu surgimento como nação é marcado por valores liberais e individualistas presentes na constituição. Estudos históricos atribuem a vitória na Guerra Civil de um nacionalismo do tipo cívico com elementos culturais e econômicos ${ }^{10}$. Mais recentemente, alguns movimentos, políticas e acordos internacionais caracterizaram aspectos 
nacionalistas no governo americano do século passado.

O slogan America First tem origem em um movimento anti-intervencionista que se iniciou em 1917, no período entre a Primeira e a Segunda Guerra Mundial. O presidente democrata Woodrow Wilson citou esse lema para caracterizar a neutralidade dos EUA na Primeira Guerra Mundial. Também foi uma das escolhas de slogan na campanha presidencial do senador republicano Wilson Harding, na eleição subsequente que ganhou, além do Back to Normalcy. Posteriormente isso ganhou mais atenção quando o American First Committee em 1940 defendeu o isolacionismo, contra a entrada americana na Segunda Guerra, perdendo relevância logo após o ataque a Pearl Harbor ${ }^{11}$. De forma geral, observa-se que o conceito era amplamente usado por diferentes linhas ideológicas, tanto socialistas como conservadores.

A preferência nacional em compras governamentais correspondeu a outro instrumento da política nacionalista americana. $\mathrm{O}$ primeiro ato desse tipo nos EUA foi o Buy American em 1933. Este determinava uma margem de preferência de $25 \%$ para produtos produzidos nos EUA, com possibilidade de exceção devido à indisponibilidade de produto, quantitativo ofertado ou em países como Canada e Israel, pelo Acordo Geral de Tarifas e Comércio (GATT). Outro ato de preferência de compras nacionais, o Buy America Act de 1982, faz parte do Ato de Assistência ao Transporte de Superfície, definindo que compras governamentais de transporte em massa devem dar preferência de produtos domésticos ou financiados parcialmente por fundos federais ${ }^{12}$.

O acordo de Bretton Woods, em 1944, com a criação de instituições como o Fundo Monetário Internacional e o Banco Mundial, foi importante na cooperação internacional, e também de grande relevância para a liderança americana; pois propiciou vitória dos interesses nacionalistas americanos sobre uma governança multilateral globalizada. Esse privilégio se perpetua até hoje, mesmo após os EUA abandonarem a conversão do dólar ao ouro em 1971, por pressões internacionais, além de inflação e déficits ${ }^{13}$.

Por conseguinte, os governos da segunda metade do século passado foram marcados por uma liderança global da Pax Americana. Esse novo excepcionalismo americano ficou ligado à hegemonia militar e política, como exceção à normativa europeia, retomado principalmente por Reagan com promessas de uma missão americana de liderar o mundo em posição diferenciada de outras nações. Em especial, essa posição de cunho cívico religiosa é marcada por uma baixa tendência à cooperação internacional sem percepção de ganhos nesse sentido ${ }^{14}$.

Na saúde, apesar de Salk ter determinado sua descoberta da vacina de pólio livre de patente, a produção comercial criou preços abusivos para a vacina nos EUA, e problemas na produção de um laboratório licenciado resultaram em 120.000 doses de vírus ativo com milhares de sequelas e algumas mortes ${ }^{15}$. Após isso a vacinação foi promovida gratuitamente pelo governo, apesar do grande receio; e os EUA não apresentam casos de Pólio desde 1979, muito antes de outros países ${ }^{16}$. Em outro episódio, alertado sobre uma possível epidemia de gripe suína em 1976, Gerald Ford acelerou o processo de produção vacinando 40 milhões de pessoas, com alguns efeitos adversos de síndrome de Guillain-Barré ${ }^{17}$.

O Plano Presidencial Emergencial para Alívio da Aids (PEPFAR) do presidente Bush, em 2003, consolidou a liderança mundial americana no mercado farmacêutico após garantir o abastecimento doméstico ${ }^{18}$. Em 2005, em resposta à possível epidemia de gripe aviária H5N1, Bush solicitou US $\$ 7,5$ bilhões para resposta, dos quais 1 bilhão para a compra de Oseltamivir e 1,2 bilhão para vacinas ${ }^{19}$.

No governo Obama, foram aprovados US $\$ 7,65$ bilhões destinados ao combate à gripe suína em 2009, sendo 1 bilhão para a compra de vacinas ${ }^{20}$. Com uma promessa inicial de 100 milhões de doses das vacinas, um problema de manufatura reduziu a previsão para 40 milhões, mas de fato, em outubro do mesmo ano, apenas 11 milhões de doses estavam disponíveis ${ }^{21}$. No ano seguinte, após terem garantido a cobertura nacional, os EUA, assim como outros países de renda alta, doaram $10 \%$ do estoque de vacinas aos outros países.

Em que pese o ideário nacionalista recente, Obama foi o primeiro presidente a ter que se posicionar publicamente sobre o excepcionalismo americano, tendo sido criticado por suas posições multilaterais ${ }^{22}$. Por sua vez, Trump mencionou o slogan America First em várias oportunidades e no seu discurso de posse.

Embora o Partido Republicano tenha sido a favor da globalização por um longo período, isso se inverteu com Trump, concomitante à mudança no apoio do Partido Democrático ao acordo Trans-Pacific Partnership (TPP) ${ }^{23}$. Ao longo do atual governo, os EUA se retiraram de acordos internacionais como o TPP, e também anunciaram a saída da $\mathrm{OMS}^{24}$. Recentemente, em agosto de 2020, Trump assinou uma ordem presidencial demandando que medicamentos essenciais 
e produtos para saúde comprados pelo governo sejam fabricados nos EUA, sendo o FDA responsável por estabelecer essa lista de produtos.

Voltando a atenção para a eleição americana em 2020, os interesses nacionalistas permanecem constantes, quando o candidato democrata Joe Biden também apresenta esse aspecto em seu plano Made in All of America com propostas de Buy American, Make it in America, Innovate in America e Supply America ${ }^{25}$. Apesar disso, o candidato se apresenta como mais favorável a uma liderança americana nas cooperações globais.

\section{Resposta Federal dos EUA à pandemia}

O principal órgão federal de saúde nos EUA é o departamento de Saúde e Serviços Humanos (HHS -Health and Human Services), equivalente ao Ministério da Saúde em muitos países. Incialmente criado em 1939 como Serviço de Saúde Pública, o HHS ainda mantém esse componente na sua estrutura, parte da qual contém o Centers for Disease Control and Prevention (CDC), o National Institutes of Health (NIH) e o Food and Drug Administration (FDA). Atualmente, com especial relevância na pandemia, a divisão Biomedical Advanced Research and Development Authority (BARDA) criada em 2006, responde ao Gabinete do Secretário Adjunto para Preparação e Resposta.

Logo no início da pandemia, a resposta dos EUA apresentou falhas. Em um primeiro episódio nacionalista os EUA optaram por um teste próprio em vez do utilizado globalmente. O teste do CDC apresentou falhas com uma das sondas, conforme relatado por diversos laboratórios oficiais dos EUA ${ }^{26}$ Apenas em 19 de agosto, o HHS rescindiu a necessidade de aprovação do FDA para testes ${ }^{27}$.

Outro episódio que atrasou a testagem nos EUA tem a ver com a declaração do Estado de Emergência pelo Secretário de Saúde americano em 31 de janeiro de $2020^{28}$. Geralmente permite-se que laboratórios de instituições usem testes próprios em seus domínios. No entanto, após a implementação do Pandemic and All-Hazards Preparedness Reauthorization Act de 2013, o Congresso Americano mudou a seção 564 do Federal Food, Drug and Cosmetic (FDC Act) para delegar Autorização de Uso Especial ao FDA em caso de doenças infecciosas emergentes, visando uma maior segurança nos testes ${ }^{29}$.

O primeiro grande ato no orçamento em vigor foi o Coronavirus Preparedness and Response Supplemental Appropriations Act em 6 de março, que liberou US\$8,3 bilhões para resposta à COVID-19. Do total, o projeto de lei outorgou mais de US\$3 bilhões para pesquisa e desenvolvimento de testes diagnósticos, terapias e vacinas, embora apenas US\$300 milhões destinados à aquisição deles. Em especial, a lei determinou um montante de US\$1,25 bilhão para assistência internacional, em programas diplomáticos, de saúde pública global, assistência a desastres ou suporte econômico ${ }^{30}$. Ao final de março, o Coronavirus Aid, Relief, and Economic Security Act (CARES $A c t)$ destinou um maior suporte econômico, de US\$2,2 trilhões, para indivíduos, empresa, governos locais, educação e saúde, sendo US\$135 bilhões destinados aos cuidados em saúde ${ }^{31}$.

O NIH, principal agência americana para o financiamento de pesquisa biomédica ou de saúde pública, é responsável por cerca de $28 \%$ de um total de US\$100 bilhões de investimentos anuais em pesquisa biomédica nos EUA. Ambos os orçamentos do NIH e do CDC aumentaram em valores reais desde sua criação em 1962. Destaca-se que o montante para pesquisa, financiado pelo NIH, passou de US\$ 15 bilhões em 1990 até quase US $\$ 40$ bilhões em 2010, se mantendo relativamente estável em cerca de US\$30 a 35 bilhões nos últimos anos, com decréscimo real descontada a inflação ${ }^{32}$. No entanto, ao longo dos anos, como parcela do orçamento do departamento de Health and Human Services (HHS) ele apresenta uma tendência de redução, em especial após a criação em 1965, do Medicare e do Medicaid.

No total em nível federal, cerca US\$3,5 trilhões são gastos com saúde por ano, isso equivale a aproximadamente $5 \%$ do PIB dos EUA. Conforme apontado na Figura 1, o HHS teve um orçamento de US\$1,2 trilhão em 2019, e desde 2010 mais de $85 \%$ dos recursos são gastos com o componente federal de assistência (valor superior a US\$1 trilhão em 2019, apenas com os programas Medicare e Medicaid).

Como consequência dos ataques com Antrax que aconteceram após o de 11 de setembro de 2001, os EUA criaram um organismo de defesa biomédica, a BARDA, a partir do Ato de Preparo a Pandemias ${ }^{33}$. Sua estrutura surge do Projeto Bioshield ${ }^{34}$, que em 2004 previa a compra de US\$5 bilhões em vacinas contra ataques bioterroristas. A missão da BARDA é "desenvolver e adquirir contramedidas médicas necessárias, incluindo vacinas, terapêuticas, diagnósticos e contramedidas não farmacêuticas, contra uma ampla gama de ameaças à saúde pública, sejam de origem natural ou intencional".

Desde então o orçamento anual da BARDA se manteve em cerca de US\$1 bilhão; financiando tecnologias médicas para prevenir ataques terroristas ou emergências de saúde pública, assim 
como estoques estratégicos. Nesse sentido, além de Antrax, a BARDA investiu em produtos contra botulismo, influenza, sarampo, ebola, zika, gases mostarda, cloro, e eventos nucleares. Para a COVID-19, o órgão diversificou as medidas procurando parcerias para produtos e capacidade de manufatura, conforme Figura 2 e Tabela 1, respectivamente. Nos produtos, o maior destaque é para vacinas, correspondendo a US\$ 10,7 bilhões do total de quase US\$ 12 bilhões. Não sendo observadas novas previsões de investimentos para vacinas desde o início de agosto, os investimentos seguem em diagnóstico, capacidades de resposta rápida (como diagnósticos de sepse e sensores remotos) e manufatura.

Embora a resposta americana tenha apresentado falhas iniciais na produção de testes diagnósticos, sobretudo na coordenação de medidas não farmacológicas, atualmente a resposta conta com grande volume de recursos para financiamento de produtos. A Operação Warp Speed, com a participação de diversas instituições, é um programa de parcerias público-privadas que visa acelerar o desenvolvimento, manufatura e distribuição de diagnósticos, terapias ou vacinas para COVID-19. Apesar da estratégia abrangente, seu principal objetivo é produzir e entregar $300 \mathrm{mi}-$ lhões de doses de vacinas, e nesse sentido, 7 candidatos mais promissores a vacinas foram selecionados em maio.
Quando anunciada em abril de 2020, a Operação Warp Speed teria mais de US\$10 bilhões à disposição, sendo inicialmente 6,5 bilhões para BARDA e 3 bilhões para o NIH. Desde então foram aportados diversos recursos na forma de AMCs, muitos deles dependendo de resultados de testes clínicos com sucesso ${ }^{35}$. Para comandar a operação foram convidados Moncef Slaoui e o general Gustave Perna, esse último com experiência logística no Comando de Materiais do Exército Américo, é diretor de operações.

Diversas instituições participam da Operation Warp Speed: alguns componentes do HHS, como o NIH, BARDA, CDC, FDA, além do Departamento de Defesa, Agricultura, Energia e de Assuntos dos Veteranos (Veteran Affairs). Essa articulação foi inspirada em medidas contra epidemias anteriores como $\mathrm{Zika}^{36}$. Um organograma da operação indica a forte presença de militares no comando, principalmente na área de operações, embora sinalize a presença de alguns profissionais de saúde pública como o chefe do CDC, além de representantes do BARDA e do $\mathrm{NIH}^{37}$. Conforme evidenciado pela Tabela 2, os aportes relatados no sítio do HHS sobre a operação Warp Speed correspondem aos investimentos da BARDA, incluindo alguns investimentos em manufatura e um aporte para anticorpos. As doses de vacinas correspondem apenas a pagamentos já comprometidos, podendo haver demandas adicionais previstas.

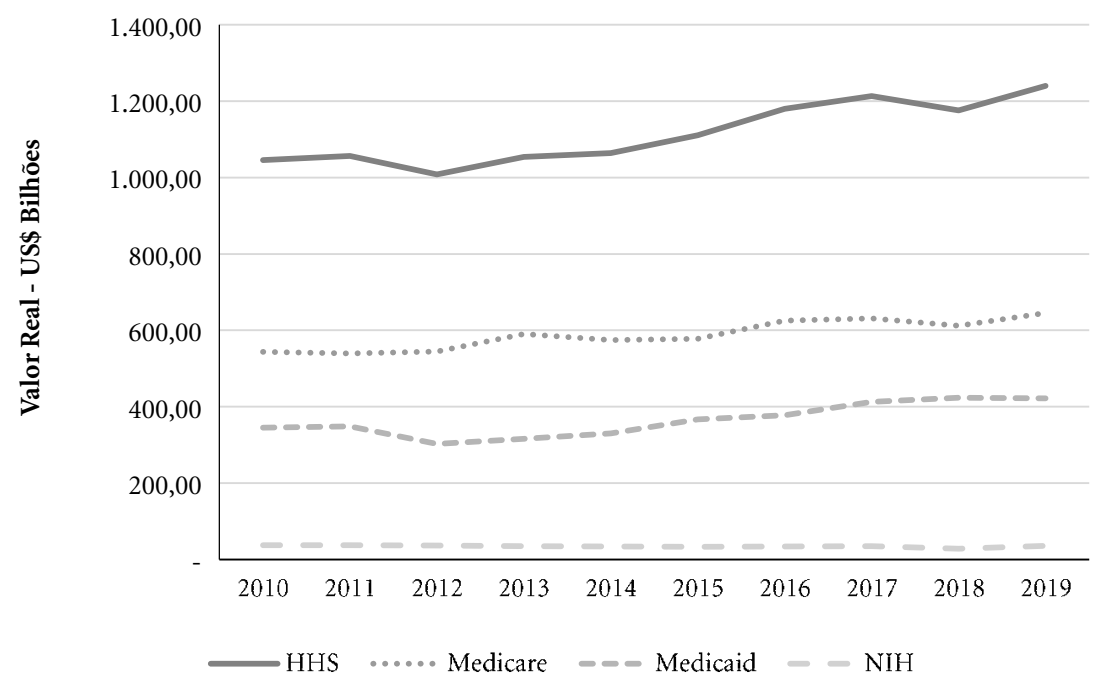

Figura 1. Histórico de Componentes do Orçamento do HHS. 
Com os recursos da operação Warp Speed, o NIH-Wide Strategic Plan for Covid 19 Research definiu 5 prioridades: conhecimento fundamental, detecção, tratamento, prevenção e disparidades em populações vulneráveis. Dois programas principais implementam isso, o Accelerating $\mathrm{CO}$ VID-19 Therapeutic Interventions and Vaccines (ACTIV) para acelerar tratamentos e vacinas e o
Rapid Acceleration of Diagnostics (RAD-X) para diagnósticos. A maioria dos recursos foram alocados no National Institute of Allergy and Infectious Diseases (NIAID), um dos institutos que compõem o NIH. Dessa forma, conforme apontado na Figura 3 além do financiamento regularmente programado, a Operação Warp Speed ampliou significativamente os investimentos para

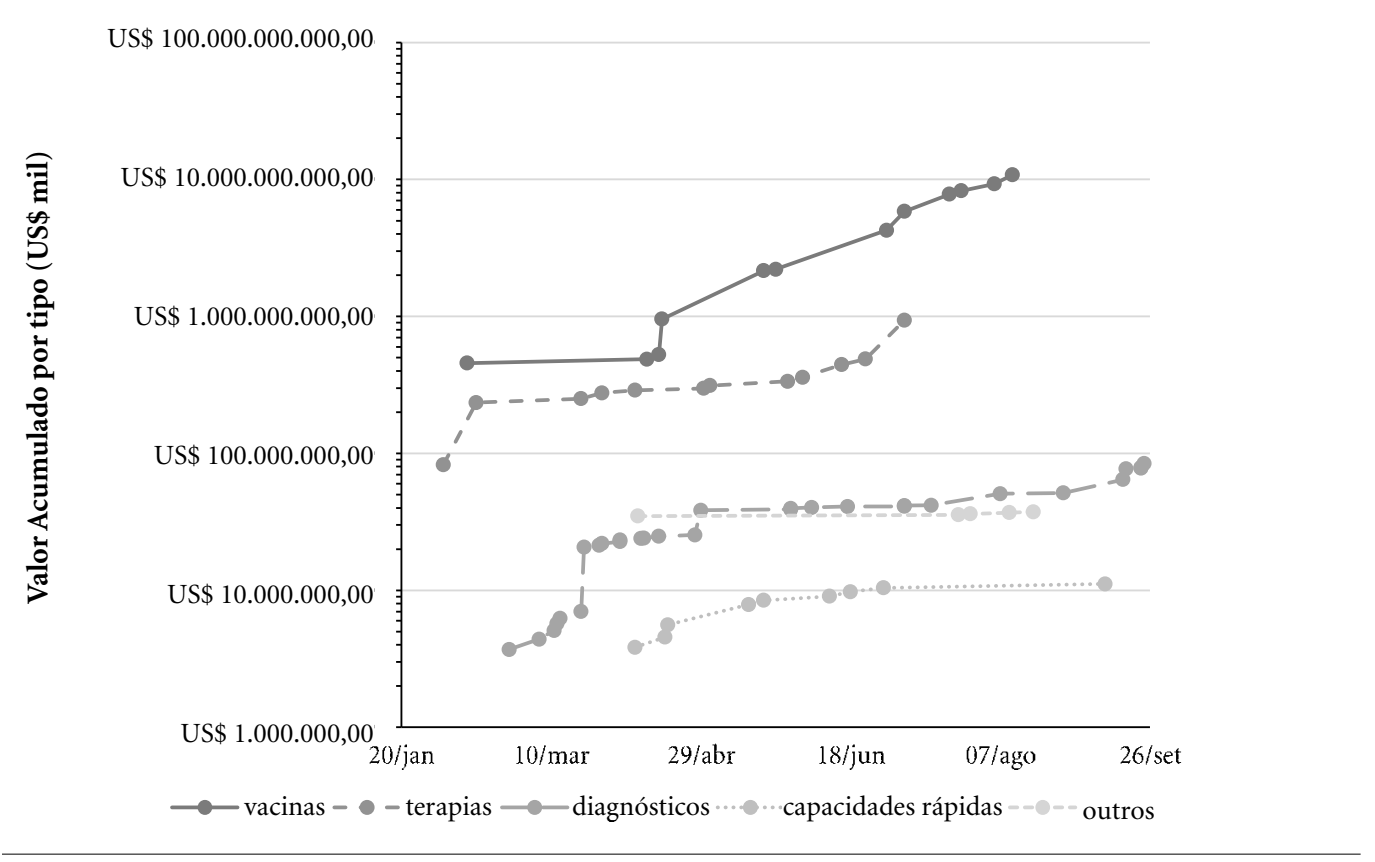

Figura 2. Valores do Portfólio da BARDA por Tipo de Produto para COVID-19.

Fonte: MedicalCountermeasures.gov (elaboração própria), acessado em 26/9/2020.

Tabela 1. Investimentos em Manufatura da BARDA para COVID-19.

\begin{tabular}{lllc}
\hline \multicolumn{1}{c}{ Data } & \multicolumn{1}{c}{ Tipo } & \multicolumn{1}{c}{ Empresa } & Valor (US\$ mil) \\
\hline $29 / 5$ & Inovação & SnapDragon Chemistry Inc. & 691,88 \\
$30 / 5$ & Doméstica & Emergent BioSolutions & $628.250,00$ \\
$05 / 6$ & Frasco & Corning Pharmacetical Tech. & $204.000,00$ \\
$05 / 6$ & Frasco & SiO2 USA & $143.000,00$ \\
$13 / 6$ & Envase & ThermoFisher Scientific & $49.189,40$ \\
$23 / 6$ & Inovação & Colorado State University & 699,99 \\
$01 / 7$ & Seringa & Retractable Technologies & $53.664,29$ \\
$01 / 7$ & Seringa & Becton, Dickson and Co. & $42.303,23$ \\
$11 / 7$ & Seringa & Smiths Medical Inc & $20.663,77$ \\
$24 / 7$ & Doméstica & Texas A\&M University & $264.693,06$ \\
$06 / 8$ & Inovação & Grand River Aseptic Manuf. & $1.600 .000,00$ \\
$06 / 8$ & Doméstica & Emergent BioSolutions & $30.000,00$ \\
$27 / 8$ & Doméstica & Ology Bioservices & $106.300,00$ \\
Total & & & $3.143 .455,62$ \\
\hline Fonte: Pharmaceutical Manufacturing in America em MedicalCountermeasures.gov (elaboração própria), acessado em 24/09/2020.
\end{tabular}




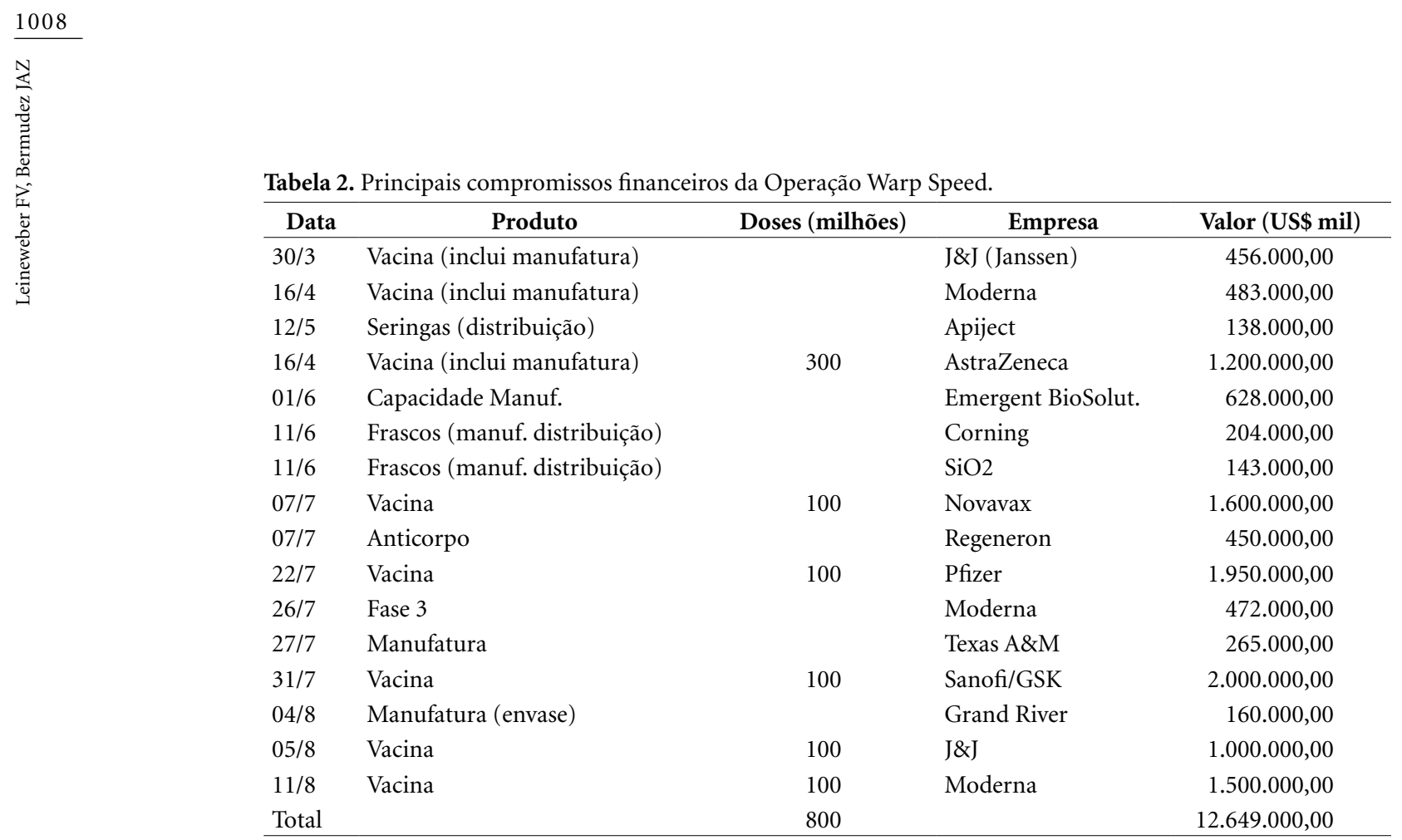

Fonte: Fact Sheet: Explaining Operation Warp Speed no hhs.gov (elaboração própria), atualizado em 24/09/2020.

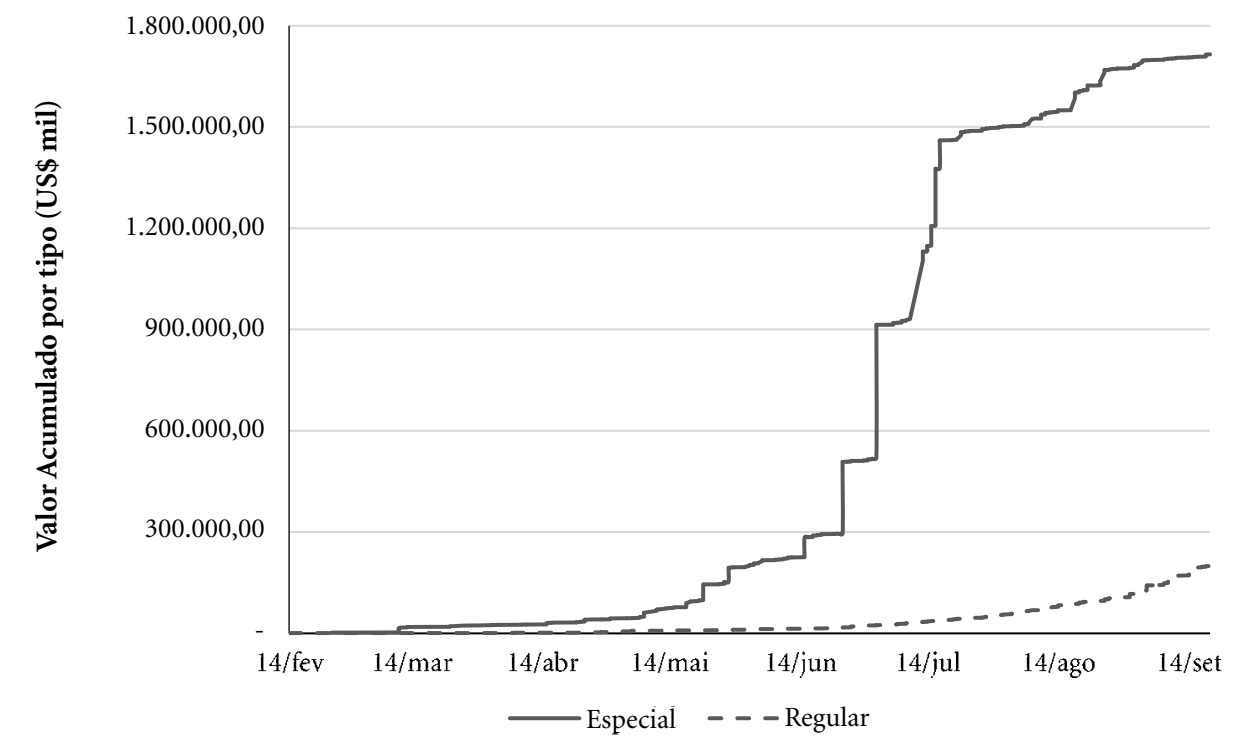

Figura 3. Investimentos de Pesquisa em COVID-19 do NIH.

Fonte: NIH Project RePORTER (elaboração própria), atualizado em 19/9/2020.

pesquisas em COVID-19. Até 19 de setembro de 2020, cerca de US\$1,7 bilhões foram investidos com recursos extraordinários e aproximadamente US\$ 300 milhões com recursos regulares ${ }^{38}$.

\section{Discussão}

Embora tenha liderado o movimento para a Declaração Universal dos Direitos Humanos, os EUA se retiraram do Conselho na ONU e se opu- 
seram à declaração nos anos 1950, tendo ratificado alguns tópicos após os anos 1960. A sociedade americana se identifica mais com valores como liberdade individual e democracia, conforme observado nas suas justificativas em intervenções internacionais. Destaca-se assim que o excepcionalismo americano legitima a posição hegemônica, assim como o america first. Dessa forma, a retomada de valores nacionalistas na identidade americana viabiliza atitudes isolacionistas.

Isto posto, observa-se que os EUA não assinaram o acordo global para vacinas Covax, e dessa maneira se deslocam do papel de liderança mundial que assumiram nas últimas décadas. Em um aspecto amplo, governos sentem uma pressão em responder a seus próprios cidadãos. Mas a resposta americana tem como base valores políticos alinhados com um já enorme volume de recursos gastos por saúde no ano, ambos aproveitando da confiança na moeda como base de referência mundial; uma disponibilidade de capital externo que demanda investimentos. Consequentemente, alavancar a capacidade inovadora em saúde se torna oportuna.

Nas iniciativas multilaterais ou nacionais, o mecanismo mais utilizado para garantir o fornecimento de diagnósticos, terapias ou vacinas para COVID-19 são os Advanced Market Commitments, com ou sem compartilhamento de custos, além de investimentos diretos ou indiretos. Deve-se destacar que muitas das empresas americanas destinatárias de recursos da Warp Speed também foram financiadas previamente por mecanismos de cooperação global. As plataformas da Moderna, Curevac e Innovio receberam investimento da Coalition for Epidemic Preparedness Innovations, assim como a Universidade de Oxford $^{39}$. Esses desenvolvimentos anteriores para a Middle Eastern Respiratory Syndrome (MERS), foram adaptados para COVID-19 pela similaridade, devido ambos serem coronavirus. Em outro aspecto semelhante, o governo americano concordou em pagar US\$2.340 por tratamento com Remdesivir (US\$390 por frasco), tendo comprado $90 \%$ da produção total por 3 meses, totalizando 500.000 frascos. No entanto, estimativas de investimento do governo americano na P\&D desse produto vão de pelo menos US $\$ 70,5$ milhões ${ }^{40}$ a US\$6,5 bilhões, este último levando em conta diversas pesquisas financiadas pelo $\mathrm{NIH}^{41}$. Por demais, a valorização de ações das empresas conforme resultados ou rodadas de investimentos é significativa.

O relatório de 2019 Global Health Security Index ${ }^{42}$ colocava os EUA como o país mais pre- parado para resposta a uma pandemia. Porém, ao abdicar de muitos meios de resposta em favor de instâncias políticas, a resposta americana à pandemia reforçou a responsabilidade individual nas escolhas dos riscos de comportamentos, motivando desconfiança na população. Pesquisa recente demonstra que a maioria dos americanos não apoia saúde universal, no entanto apoiam produção pública e licenciamento de medicamentos $^{43}$. As respostas políticas compartilham esse ideário evidenciado nas precificações; onde a saúde está contaminada por uma narrativa econômica em lugar de direitos.

A evolução da resposta global remete influências no âmbito externo. Os países mais ricos, com $13 \%$ da população mundial asseguraram mais de $51 \%$ das doses previstas de vacinas ${ }^{44}$. Os EUA conseguiram assegurar cerca 800 milhões de doses de vacinas, com potencial para aquisição mais de 1,5 bilhão, previsto nos acordos. Considerando sua grande população, isso é inferior ao garantido per capita pelo Reino Unido, e próximo ao disposto pela União Europeia ${ }^{45}$. Poucos países periféricos dispõem de tal acesso potencial, ainda mais exacerbado se considerada a probabilidade de sucesso na aprovação de vacinas em desenvolvimento.

O arranjo institucional apresentado também revela um aspecto interno excepcional dos investimentos produtivos. Apesar de ter historicamente muito pouca manufatura de vacina em comparação com a Europa ${ }^{46}$, os EUA garantiram 4 bilhões de doses a serem fabricadas no seu território, enquanto outros países com maior capacidade prévia, como Reino Unido e Índia, fabricariam cerca de 1,5 bilhão de doses cada um $^{45}$. Ademais, ao confrontar os esforços nacionalistas com protagonismo nas vacinas, é necessário ampliar para além dos outros produtos na prevenção ou para o combate à COVID-19, evidenciado pelos recursos em pesquisa do NIH, com a possível repercussão dos valores das inovações americanas no acesso global a tecnologias em saúde.

O nacionalismo compete com iniciativas solidárias. Medidas globais devem ser complementadas por inciativas regionais coordenadas, com respectivas adequações nas políticas nacionais. Esse tipo de colaboração seria uma resposta mais adequada para a pandemia, considerando a interconectividade mundial. No entanto, os EUA buscam caminhos independentes, com base em ideários nacionalistas viabilizados por um forte complexo econômico-industrial de saúde. Por conseguinte, o nacionalismo também se propaga por diversos países do mundo, seja nas medidas 
protecionistas, disputas por influência ou preferências locais, como no caso recente da Comissão Europeia, que declarou interesse em ter um organismo semelhante ao americano, citando explicitamente a BARDA ${ }^{47}$.

Considerando a multiplicidade de tecnologias para respostas à pandemia, o desequilíbrio entre oferta e demanda e a dependência podem afetar países periféricos com menor poder econômico como o Brasil. A alocação de recursos econômicos para as inovações, precipuamente direcionados às vacinas, acirra a preferência temporal de países centrais. $\mathrm{O}$ acesso às tecnologias inclui disponibilidade efetiva no tempo adequado, mas a capacidade de financiamento causa uma defasagem que se acirra na pandemia.

A conceituação imprecisa da formação de cadeia de soluções que se tornam tardiamente acessíveis, reflete a natureza complexa, transdisciplinar e multifacetada da Saúde Coletiva. Dessa forma, a incoerência entre os interesses econômicos refletidos na identidade nacional e os direitos humanos, desafia a Saúde Coletiva não apenas no direito à saúde, mas também nas relações internacionais com seus arranjos na geopolítica contemporânea.

\section{Considerações finais}

Desde o início da pandemia, o mundo se viu envolvido em uma série de iniciativas de solidariedade descritas na introdução deste artigo, objetivando assegurar acesso às tecnologias relacionadas com o diagnóstico, prevenção, tratamento e proteção das populações, focalizando na necessidade de expandir essas iniciativas aos países com menos recursos e às populações negligenciadas e vulneráveis. Diversos países também estabeleceram alterações nos seus marcos regulatórios para facilitar esse tipo de acesso. Entretanto, a logística e organização presentes na resposta dos EUA, ao não se somar com nenhuma iniciativa de caráter global ou regional, e com raízes em momentos históricos anteriores, podem gerar hiatos de desabastecimento no processo de competição acirrada que foi colocada em prática com as diretrizes atuais. Fica evidente que essa iniciativa pode provocar um desequilíbrio entre oferta e demanda de tecnologias em nível mundial.

Em suma, o nacionalismo financeiro viabilizou uma resposta atendendo mais a interesses econômicos do que à saúde. O prestígio da coordenação de uma resposta nacional limitada a interesses comerciais, exclui uma efetiva solidariedade na resposta à pandemia. Apesar dos valores dos fundadores dos EUA considerarem a liberdade individual, a perspectiva coletiva do direito universal à saúde depende do acesso aos produtos considerados bens públicos. $\mathrm{E}$ a utilização disso como um bem político permanece sempre como outra ameaça, que pode excluir populações negligenciadas e vulneráreis, prejudicando repostas globais a essa pandemia e outras condições para as quais a solidariedade é necessária.

\section{Colaboradores}

Ambos os autores participaram do desenvolvimento deste artigo original. FV Leineweber participou no levantamento de referências, pesquisa de dados e elaboração do tema, enquanto JAZ Bermudez colaborou na concepção, desenvolvimento e redação final. 


\section{Referências}

1. Guimarães R. Vacinas Anticovid: um Olhar da Saúde Coletiva. Cien Saude Colet 2020; 25(9):3579-3585.

2. Guimarães R. As Interfaces e as "Balas de Prata": Tecnologias e Políticas. Cien Saude Colet 2020; 25(9):3563-3566.

3. Bermudez J, Leineweber FV. "Tecnologias de Saúde - Medicamentos e Vacinas: Bens Públicos Globais ou Disputa de Mercado". In:Buss PM, Fonseca LE, organizadores. Diplomacia Da Saúde e a Pandemia: Reflexões a Meio Do Caminho. Rio de Janeiro: Editora Fiocruz; 2020.

4. Briana J, Bourland S. Companies Keep Raising Prices in the Midst of a Pandemic [Internet]. Patients for Affordable Drugs; 2020 [acessado 2020 Set 9]. Disponível em: https://patientsforaffordabledrugs. org/2020/06/28/covid-price-hikes-report/

5. Augustovski F, McClellan MB. Current Policy and Practice for Value-Based Pricing. Value Health. 2019; 22(6):S4-S6.

6. Health and Human Services (HHS) Aquisiton Regulation [Internet]. HHS. Sect. 335; 2015 [acessado 2020 Set 9]. Disponível em: https://www.hhs.gov/grants/ contracts/contract-policies-regulations/hhsar/part335-research-development-contracting/index.html

7. Berndt ER, Glennerster R, Kremer MR, Lee J, Levine R, Weizsäcker G, Williams H. Advance market commitments for vaccines against neglected diseases: estimating costs and effectiveness. Health Economics 2007; 16(5):491-511.

8. Hickey KJ, Ward EH. Legal Issues in COVID-19 Vaccine Development [Internet]. Congressional Research Service; 2020 [acessado 2020 Set 9]. Disponível em: https://crsreports.congress.gov/product/pdf/R/ R46399

9. Government Accountability Office (GAO). COVID-19 Federal Efforts Could Be Strengthened by Timely and Concerted Actions [Internet]. United States GAOe; 2020 [acessado 2020 Set 23]. Disponível em: https://www.gao.gov/assets/710/709492.pdf

10. Lepore J. A new Americanism: Why a nation needs a national story [Internet]. Foreign Affairs. 2019 [acessado 2020 Set 10]; 98(10). Disponível em: https://www. foreignaffairs.com/articles/united-states/2019-02-05/ new-americanism-nationalism-jill-lepore

11. Kupchan C. The Clash of Exceptionalisms. Foreign Affairs 2018; (97):138.

12. Frank D. Buy American the untold story of economic nationalism. Boston: Beacon Press; 2005.

13. Carter ZD. The price of peace: money, democracy, and the life of John Maynard Keynes. New York: Random House; 2020.

14. Sachs J. A new foreign policy: beyond American exceptionalism. New York: Columbia University Press; 2018.

15. Offit PA. The Cutter Incident, 50 Years Later. $N$ Engl $J$ Med. 2005; 352(14):1411-1412.

16. Ochmann S, Roser M. Polio [Internet]. Our World in Data; 2017 [acessado 2020 Set 20]. Disponível em: https://ourworldindata.org/polio

17. Sencer DJ, Millar JD. Reflections on the 1976 Swine Flu Vaccination Program. Emerg Infect Dis. 2006; 12(1):23-28
18. Jadeja S, Pai G, Bhat K, Sathyanarayana MB. President's Emergency Plan for AIDS Relief. SRP 2018; 9(1):6-9.

19. Bush GW. President Outlines Pandemic Influenza Preparations and Response [Internet]. Bush White House Archives; 2005 [acessado 2020 Set 28]. Disponível em: https://georgewbush-whitehouse.archives.gov/news/ releases/2005/11/20051101-1.html

20. Cohen J. House Approves \$8 Billion for Swine Flu Pandemic [Internet]. Science; 2009 [acessado 2020 Set 28]. Disponível em: https://www.sciencemag.org/ news/2009/06/house-approves-8-billion-swine-flu -pandemic

21. Korecki N. Biden has fought a pandemic before. It did not go smoothly [Internet]. Politico; 2020 [acessado 2020 Set 28]. Disponível em: https://www.politico. com/news/2020/05/04/joe-biden-contain-h1n1-virus-232992

22. Obama B. Remarks by the President Delivers at the Global Health Security Agenda Summit [Internet]. Obama White House Archives; 2014 [acessado 2020 Set 14] Disponível em: https://obamawhitehouse.archives. gov/node/300511

23. Kamarck E, Podkul A. Role reversal: Democrats and Republicans express surprising views on trade, foreign policy, and immigration [Internet]. Brookings; 2018 [acessado 2020 Set 10]. Disponível em: https://www. brookings.edu/blog/fixgov/2018/10/25/role-reversaldemocrats-and-republicans-express-surprising-views -on-trade-foreign-policy-and-immigration/

24. Dujarric S. Note to Correspondents in answer to questions regarding the World Health Organization [Internet]. United Nations Secretary-General; 2020 [acessado 2020 Set 10]. Disponível em: https://www.un.org/ sg/en/content/sg/note-correspondents/2020-07-07/ note-correspondents-answer-questions-regardingthe-world-health-organization

25. Biden J. The Biden Plan to Ensure the Future is "Made in All of America" by All of America's Workers [Internet]. Joe Biden for President: Official Campaign Website; 2020 [acessado 2020 Set 14]. Disponível em: https://joebiden.com/made-in-america/

26. Cohen J. The United States badly bungled coronavirus testing — but things may soon improve [Internet] Science; 2020 [acessado 2020 Set 10]. Disponível em: https://www.sciencemag.org/news/2020/02/united-states-badly-bungled-coronavirus-testing-things-may-soon-improve

27. Health and Human Services (HHS), Assistant Secretary for Public Affairs (ASPA). Rescission of Guidances and Other Informal Issuances [Internet]. HHS; 2020 [acessado 2020 Set 10]. Disponível em: https://www. hhs.gov/coronavirus/testing/recission-guidances-informal-issuances-premarket-review-lab-tests/index. html

28. Health and Human Services (HHS), News Division Secretary Azar Declares Public Health Emergency for United States for 2019 Novel Coronavirus [Internet]. HHS; 2020 [acessado 2020 Set 10]. Disponível em: https://www.hhs.gov/about/news/2020/01/31/secretary -azar-declares-public-health-emergency-us-2019-novel-coronavirus.html 
29. Food and Drug Administration (FDA), Emergency Use Authorization [Internet]. FDA Office of the Commissioner; 2020 [acessado 2020 Set 10]; Disponível em: https://www.fda.gov/emergency-preparedness-and-response/mcm-legal-regulatory-and-policy-framework/emergency-use-authorization

30. Coronavirus Preparedness and Response Supplemental Appropriations Act. Pub. L. No. 116-123, 116th Cong. Jun 3, 2020.

31. Coronavirus Aid, Relief, and Economic Security Act (CARES Act) Sect. 3548, 116ht Cong. Mar 6, 2020.

32. Health and Human Services (HHS), Assistant Secretary for Financial Resources (ASFR) [Internet]. Office of Budget (OB); 2019 [acessado 2020 Set 14]. Disponível em: https://www.hhs.gov/about/budget/index. html

33. Pandemic and All-Hazards Preparedness Act. Sect. 3678, Pub. L. No. 109-417, 109th Cong., 2006.

34. Project BioShield Act. Sect. 15, Pub. L No. 108-276, 108th Cong., 2004.

35. Health and Human Services (HHS). Fact Sheet: Explaining Operation Warp Speed [Internet]. HHS; 2020 [acessado 2020 Set 20]. Disponível em: https://www. hhs.gov/coronavirus/explaining-operation-warp-speed/index.html

36. Slaoui M, Hepburn M. Developing Safe and Effective Covid Vaccines - Operation Warp Speed's Strategy and Approach. N Engl J Med 2020; 383(18):17011703.

37. Florko N. New chart reveals military's vast involvement in Operation Warp Speed [Internet]. Stat News; 2020 [acessado 2020 Set 29]. Disponível em: https://www. statnews.com/2020/09/28/operation-warp-speedvast-military-involvement/

38. National Institutes of Health (NIH). NIH-Wide Strategic Plan for COVID-19 Research [Internet]. NIH; 2020 [acessado 2020 Ago 10]. Disponível em: https:// www.nih.gov/sites/default/files/research-training/initiatives/covid-19-strategic-plan/coronavirus-strategic-plan-20200713.pdf

39. Christodoulou M. CEPI Awards Contract Worth Up To USD\$19 million to Oxford University and Janssen Vaccines to Develop MERS, Lassa, and Nipah Vaccines [Internet]. CEPI; 2018 [acessado 2020 Set 10]. Disponível em: https://cepi.net/news_cepi/cepi-awards-contrac$\mathrm{t}$-worth-up-to-usd19-million-to-oxford-university-and-janssen-vaccines-to-develop-mers-lassa-andnipah-vaccines/

40. Public Citizen. The Real Story of Remdesivir [Internet]. Public Citizen; 2020 [acessado 2020 Set 10]. Disponível em: https://www.citizen.org/article/the-real -story-of-remdesivir/
41. Cleary EG, Jackson MJ, Folchman-Wagner Z, Ledley FD. Foundational research and NIH funding enabling Emergency Use Authorization of remdesivir for COVID-19. medRxiv; 2020. doi: https://doi. org/10.1101/2020.07.01.20144576

42. Johns Hopkins Center for Health Security. The Global Health Security Index [Internet]. GHS Index; 2019 [acessado 2020 Set 17]. Disponível em: https://www. ghsindex.org/

43. Yglesias M. Poll: Fixing drug prices is more popular than Medicare-for-all [Internet]. Vox; 2020 [acessado 2020 Set 24]. Disponível em: https://www.vox. com/2020/2/24/21134677/polling-progressive-ideasmedicare-for-all-drug-costs-climate

44. Tabacek K. Small group of rich nations have bought up more than half the future supply of leading COVID-19 vaccine contenders [Internet]. Oxfam International; 2020 [acessado 2020 Set 17]. Disponível em: https:// www.oxfam.org/en/press-releases/small-group-richnations-have-bought-more-half-future-supply-leading-covid-19

45. Callaway E. The unequal scramble for coronavirus vaccines - by the numbers. Nature 2020; 584(7822):506-507.

46. Lincicome S. What if Politicians Are the Biggest Medical Supply Chain Risk? [Internet]. Cato Institute; 2020 [acessado 2020 Set 17]. Disponível em: https://www. cato.org/blog/whats-biggest-risk-facing-medical-supply-chains

47. von der Leyen U. State of the Union Address: charting the course out of the coronavirus crisis and into the future [Internet]. European Commission; 2020 [acessado 2020 Set 29]. Disponível em: https://ec.europa.eu/ commission/presscorner/detail/en/ip_20_1657

Artigo apresentado em 07/10/2020

Aprovado em 21/11/2020

Versão final apresentada em 23/11/2020

Editores-chefes: Romeu Gomes, Antônio Augusto Moura da Silva 